\title{
yesThe Complexities of ART Which Prevent Durable Viral Suppression Anthony Amoroso*f
}

\author{
Address: Institute of Human Virology \\ Email: Anthony Amoroso* - amoroso@umbi.umd.edu \\ * Corresponding author ¥Presenting author
}

from 2005 International Meeting of The Institute of Human Virology

Baltimore, USA, 29 August - 2 September 2005

Published: 8 December 2005

Retrovirology 2005, 2(SuppI I):SI 23 doi: I0. I 186/I742-4690-2-SI-SI 23

The complexities of ART which prevent durable viral suppression should not be under estimated, and include the need for absolute adherence, the rapid development of resistance, and the impact of cross resistance, unequal potency of ARV's, pharmacokinetic limitations, and drug toxicities. Providers of HIV care in the U.S. are faced with the increased complexities of care as they encounter growing numbers of patients with drug resistance. Reliance on frequent use of viral load monitoring and genotypic analysis has become the recommended norms.

As the world prepares for antiretroviral scale up programs in resource limited nations, the need to achieve durable antiretroviral therapy (ART) success across multiple different patient populations and to decrease the reliance on frequent viral loads and genotypes to gauge treatment outcomes has become more apparent. Treatment programs in clinics which serve patients in resource limited settings are trying to address these complexities to improve long term treatment success. 